\title{
SEMS for Palliation of Malignant Colonic Obstruction: Why SEMi Available Is Not Good Enough
}

\author{
Christian P. Selinger ${ }^{1}$
}

Published online: 10 February 2016

(c) Springer Science+Business Media New York 2016

In the past, treatment for acute colonic obstruction was emergency surgery with ostomy. Over the last two decades, the availability of colonic self-expanding metal stents (SEMSs) has provided new treatment options and therapeutic paradigms for malignant obstruction of the colon. In the setting of incurable colorectal cancer (CRC) complicated by colonic obstruction, colonic SEMS placement is the preferred option for palliation of patients not receiving antiangiogenic therapy [1].

The main advantages of SEMS in the palliative setting are its less-invasive nature and low mortality [1]. SEMS has many advantages over surgery, including shorter postprocedural recovery times and the avoidance of general anesthesia and ostomy creation. Nevertheless, SEMS placement is not free of complications; while emergency surgery is associated with a greater risk of short-term complications, SEMS placement is associated with longterm complications such as stent migration, perforation, and recurrent obstruction $[1,2]$. Since many palliative CRC patients do not live long enough to experience these late complications, access to colonic SEMS, given its overall early advantages, is essential to the delivery of good palliative therapy in patients with incurable malignant colonic obstruction.

The original article (doi:10.1007/s10620-015-4019-8) inadvertently published in the previous issue (June 2016, Volume 61, Issue 6, pp 1669-1676)

\footnotetext{
Christian P. Selinger

Christian.selinger@web.de

1 Institute of Gastroenterology, Leeds Teaching Hospitals NHS Trust, St James University Hospital, Bexley Wing, Beckett Street, Leeds LS20 8HL, UK
}

The study by Okafor et al. [3] published in this issue of Digestive Diseases and Sciences is a timely reminder that access to colonic SEMS for palliation is not universal. By examining the National Inpatient Sample, they identified significant variations in colonic SEMS utilization among different socioeconomic groups in the USA. Patients from less affluent areas had lower utilization rates than those in more affluent areas. Furthermore, these patients waited the longest for SEMS placement as inpatients, incurring higher hospital charges [3]. There are some important limitations to the study. Radiologically placed SEMSs were not captured. The sample was limited to inpatient procedures; furthermore, indication for SEMS placement was derived from the presence or absence of metastatic disease. Hence, patients requiring palliation of malignant colonic obstruction due to their comorbid state rather than due to metastatic disease were not included.

The differences in colonic stent utilization rates reported by Okafor et al. [3] require careful examination. As access to SEMS depends on the availability of adequately trained endoscopists and/or radiologists, it is not surprising that smaller and rural hospitals used stents less often than urban teaching hospitals. Patients with commercial insurance were less likely to receive SEMS than those with Medicare, suggesting that some commercial insurers may restrict or deny access to colonic SEMS, obstacles that need to be carefully analyzed by insurers from a risk/benefit standpoint. The formation of "hub and spoke" networks between rural and teaching hospitals may facilitate access to high-quality and up-to-date procedures such as SEMS placement. In this model, "spoke" centers refer patients to "hubs" for the SEMS procedure while the "spoke" provides the initial evaluation and aftercare. This system avoids the need to train staff in colonic SEMS placement in low-volume centers unlikely to maintain proficiency due to 
inadequate procedure volume. Nevertheless, the success of such "hub and spoke" systems is dependent on the awareness and acceptance by medical staff of the arrangement in the "spoke" centers.

Multidisciplinary team meetings involving surgery, gastroenterology, radiology, histopathology, and oncology offer a way of formally assessing each patient with CRC. This model, widely used for all CRC patients in the UK [4], is also associated with better quality care [5]. Multidisciplinary team meetings could help facilitate awareness of and access to SEMS placement as well as offering opportunities to deliver more consistent therapeutic approaches. With regard to insurance, since SEMS placement is associated with shorter hospital stays and lower costs, commercial insurers may increase access to SEMS in the future.

While access-to-care is an important explanation for the differences in utilization rates, other factors are possible. Since patients from ethnic minorities often have more advanced disease at presentation [6, 7], Okafor et al. [3] hypothesize that they may require palliation with SEMS more frequently. While ethnic minority patients had higher SEMS utilization rates detected with univariate analysis, that effect disappeared with multivariate analysis. Nevertheless, the study findings highlight that early cancer diagnosis is a worthy goal, especially in lower socioeconomic groups that have limited access-to-care. Another impediment is dissatisfaction with the bowel preparationdependent invasive modality currently used for CRC screening, namely colonoscopy. Although a change to more convenient and less-invasive procedures may improve the acceptability of screening, noninvasive techniques including DNA analysis of fecal samples currently lack the requisite performance characteristics required for screening tests [8]. While such newer technologies may eventually be more appealing to the public, the greater challenge will be that of reaching population segments that participate less in screening programs that in turn awaits the rectification of the many negative perceptions of colonoscopy-based CRC screening methods [9].

The availability of colonic SEMS placement is not only vital for patients with obstructing, metastatic CRC; it may also become a useful quality indicator for clinical improvement programs. The recent publication of the European guidelines, which have been endorsed by the American Society for Gastrointestinal Endoscopy, clearly establish colonic SEMS as the preferred standard for palliation of malignant colonic obstruction [10], reinforcing our supposition that colonic SEMS should be available for every such patient.

\section{References}

1. Zhao XD, Cai BB, Cao RS, Shi RH. Palliative treatment for incurable malignant colorectal obstructions: a meta-analysis. World J Gastroenterol. 2013;19:5565-5574.

2. Selinger CP, Ramesh J, Martin DF. Long-term success of colonic stent insertion is influenced by indication but not by length of stent or site of obstruction. Int J Colorectal Dis. 2011;26: 215-218.

3. Okafor PN, Stobaugh DJ, Song LMWK, Limburg PJ, Talwalkar JA. Socioeconomic inequalities in the utilization of colorectal stens for the treatment of malignant bowel obstruction. Dig Dis Sci. (Epub ahead of print). doi:10.1007/s10620-015-4019-8

4. Patkar V, Acosta D, Davidson T, Jones A, Fox J, Keshtgar M. Cancer multidisciplinary team meetings: evidence, challenges, and the role of clinical decision support technology. Int J Breast Cancer. 2011;2011:831605.

5. Burton S, Brown G, Daniels IR, et al. MRI directed multidisciplinary team preoperative treatment strategy: the way to eliminate positive circumferential margins? Br J Cancer. 2006;94:351-357.

6. Robbins AS, Siegel RL, Jemal A. Racial disparities in stagespecific colorectal cancer mortality rates from 1985 to 2008 . $J$ Clin Oncol. 2012;30:401-405.

7. Fitzgerald TL, Bradley CJ, Dahman B, Zervos EE. Gastrointestinal malignancies: When does race matter? J Am Coll Surg. 2009;209:645-652.

8. Davies RJ, Miller R, Coleman N. Colorectal cancer screening: prospects for molecular stool analysis. Nat Rev Cancer. 2005;5:199-209.

9. Palmer CK, Thomas MC, von Wagner C, Raine R. Reasons for non-uptake and subsequent participation in the NHS Bowel Cancer Screening Programme: a qualitative study. Br J Cancer. 2014;110:1705-1711.

10. van Hooft JE, van Halsema EE, Vanbiervliet G, et al. Self-expandable metal stents for obstructing colonic and extracolonic cancer: European Society of Gastrointestinal Endoscopy (ESGE) Clinical Guideline. Endoscopy. 2014;46:990-1053. 